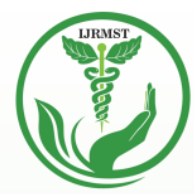

\title{
INTERNATIONAL JOURNAL OF RESEARCH IN MEDICAL SCIENCES \& TECHNOLOGY
}

\author{
e-ISSN:2455-5134; p-ISSN: 2455-9059
}

Technical Overview and Evaluation of Heartune App: Mapping

Music to Heartbeat for Recreational or Medical Purposes (Whitepaper)

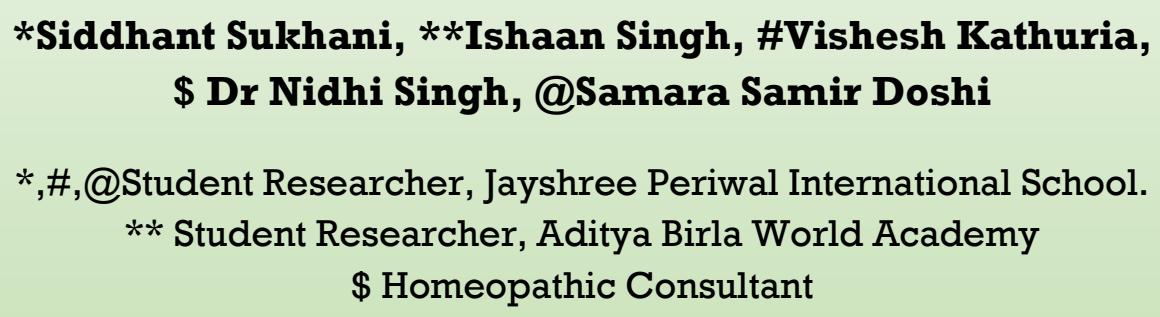

Paper Received: $08^{\text {th }}$ November, 2021; Paper Accepted: $02^{\text {nd }}$ January, 2022;

Paper Published: 05 ${ }^{\text {th }}$ January, 2022

DOI: http://doi.org/10.37648/ijrmst.v13i01.001

\section{How to cite the article:}

Siddhant Sukhani, Ishaan Singh, Vishesh Khaturia, Dr Nidhi Singh,

Technical Overview and Evaluation of

Heartune App: Mapping Music to Heartbeat for Recreational or Medical

Purposes (Whitepaper), IJRMST, January-June 2022, Vol 13, 1-26, DOI: http://doi.org/10.37648/ijrmst.v13i01.001

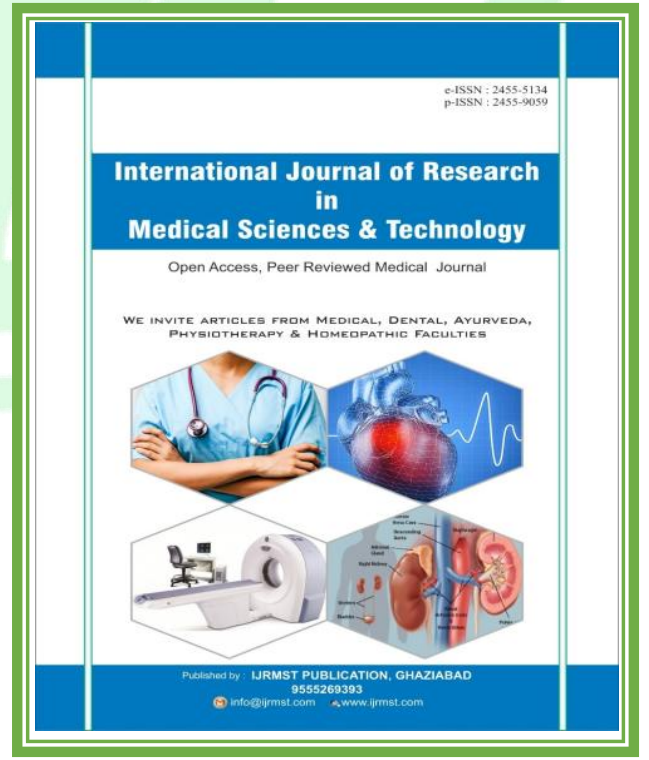




\section{VOLUME: 13, JANUARY-JUNE 2022}

\section{ABSTRACT}

This paper aims to verify the feasibility of utilizing the novel application Heartune as a supplemental treatment for Autism, Alzheimers, Depression/anxiety and developmental delays using passive music therapy. The studies and meta-analyses discussed within this whitepaper illustrate the ability of music therapy in general to benefit the wellbeing and behavioural tendencies of those diagnosed with the aforementioned illnesses, and hence the results obtained by this whitepaper justify the utilization of Heartune as a source for the supplemental treatment for over 100 million patients worldwide. Music therapy has been proven to help several children with developmental delays and Autism especially considering the behavioural changes caused by music therapy. Moreover, the therapy has led researchers to conclude that within the average duration of a song $\sim 3$ minutes, the user's heartbeat can change up to $12 \%$, indicating that the application can be utilised to change the heartbeat and hence the user's mood up to a certain extent. The concluding remarks do state that the application is complementary to the current treatment of the patients and does not in any manner propose a complete recovery from the given illness but proposes to reduce the impacts it has on the lives of the patients.

\footnotetext{
${ }^{1}$ Tuning in: How music may affect your heart - Harvard Health

"Tuning In: How Music May Affect Your Heart - Harvard Health". Harvard Health, 2018,

https://www.health.harvard.edu/heart-health/tuning-in-how-music-may-affect-your-heart.
} 


\section{INTRODUCTION}

Music therapy is a method of using musical instruments, singing or listening to music either live or through recording. Having been practiced for several years, music therapy takes on two major formsactive and passive-based on the requirements of the patient ${ }^{2}$. Active music therapy involves the patient's participation in singing alongside the therapist or playing any particular musical instrument as a form of therapeutic relief; active music therapy often involves the improvisation of music to fit the patient. On the other hand, passive music therapy, the focus of this whitepaper, usually involves having the patient listen to specific types of music live or recorded to stimulate certain parts of their brain; compared to active music therapy, passive music, passive music therapy utilises tracks chosen in advance.

Currently, the field of music therapy utilises both active and passive music therapy in various forms based on the needs of the patients ${ }^{3}$. The first of which is

\footnotetext{
2 "What Is Music Therapy?" Taking Charge of Your Health \& Wellbeing, www.takingcharge.csh.umn.edu/commonquestions/what-music-therapy.
}

\footnotetext{
${ }^{3}$ Montello, L., and E. E. Coons. "Effects of Active versus Passive Group Music Therapy on Preadolescents with Emotional, Learning, and Behavioral Disorders." Journal of Music Therapy,
}

Analytical music therapy, wherein the patient must express their subconscious thoughts by singing or playing an instrument impromptu. A more passive form of music therapy is Benenzon music therapy ${ }^{4}$ that utilises several genres of music in order to enable the patient to discover their own musical identity, therefore encouraging self-reflection. This can often be done in groups to discover a collective identity based on social relations and cultures in the form of Community music therapy, which has been practised even in the 1970s. Moreover, Cognitive Behavioural music therapy is an extension allowing for behavioural corrections in the patients. Furthermore, the Bonny Method of Guided Imagery and music involves the patient describing their imagination and emotions while listening to a specific genre of music - usually classical. More active forms of therapy include the Nordoff-Robbins - playing an instrument as a form of self-expression - and Vocal Psychotherapy - Singing music to develop a connection with oneself.

vol. 35, no. 1, 1998, pp. 49-67., doi:10.1093/jmt/35.1.49.

\footnotetext{
${ }^{4}$ Wagner, Gabriela. "The BENENZON Model of Music Therapy." Nordic Journal of Music Therapy, vol. 16, no. 2, 2007, pp. 146-147., doi:10.1080/08098130709478184.
} 
With experience in management and MedTech, the founders of Heartune aim to provide a beneficial service to over 100 million people that are currently victims of one of the following illnesses whose effects can be significantly reduced with the help of music therapy: Autism, Alzheimer's, Depression and developmental delays.

The purpose of this whitepaper is to justify the utilisation of Heartune as a form of supplemental treatment for patients with Autism, Alzheimers, Depression and developmental delays for children by referencing several studies and metaanalyses conducted previously regarding the potential benefits of using such therapeutic methods. Based on the results obtained by the researchers, the evaluation of whether an application can administer music therapy will take place alongside a general description of how the application would potentially work. Hence, this whitepaper outlines the feasibility of an application such as Heartune, the first of its kind.

\section{MUSIC AND HEARTBEAT}

The link between music and the heart rate of a given listener has been investigated for several decades, and there is a positive correlation between the two.
When investigating the entertainment aspect of the application, the relationship between preferred music types and tempos for a given heart rate is evident. As proven by Iwanaga ${ }^{5}$ in her research analysis of the correlation between music tempo and heartbeat, her study of 14 undergraduate students choosing music based was most likely to choose songs and melodies whose tempo matched their heartbeat rate. The conclusions presented by the study can hence be extrapolated to indicate that people have a strong inclination towards listening to music that aligns with one's heartbeat. Thus, the fundamental entertainment purpose of Heartune is satisfied as it would have the ability to control the music being played based on the heart rate of the listener. Moreover, using the same ideology, there is a bidirectional causal relationship between the heartbeat and the music, like the music being played also influences the heart rate of the listeners.

The inverse relationship between music and heartbeats wherein the music played influences the heartbeat was also studied

\footnotetext{
${ }^{5}$ IWANAGA, MAKOTO. "RELATIONSHIP between HEART RATE and PREFERENCE for TEMPO of MUSIC." Perceptual and Motor Skills, vol. 81, no. 2, Oct. 1995, pp. 435-440, pubmed.ncbi.nlm.nih.gov/8570336/, 10.2466/pms.1995.81.2.435. .
} 
by Agarwal et $\mathrm{Al}^{6}$. After taking a random sample of 30 high school students over the age of 18 , they conducted a series of experiments to allow the student's heart rates to reach a resting phase before playing certain slow and fast songs for 2.5 minutes. The heart rates were measured both before and after the song was played, and their experiments showed a fall in heart rate with slow music for $93 \%$ of the test group, whereas a rise in the heart rate with fast music was detected for $100 \%$ of the test group. Their results indicate a significant correlation between the heartbeat and the music played. These results hence indicate that another key feature of the application - heart rate altercations - can be administered as per the consumer's liking. Such a study further corroborates the viability of the application for both medical and entertainment purposes as not only can the application helps maintain a stable heart rate, but it can also increase or decrease the heart rate as required.

\section{HOW HEARTTUNE WORKS}

The Heartune App is intended to be used as a supplementary tool to cardiologists and music therapists. It can also be used as an individual tool for mapping music to

\footnotetext{
${ }^{6}$ JEI. "The Effect of Music on Heart Rate | Journal of Emerging Investigators." Emerginginvestigators.org, 2013, emerginginvestigators.org/articles/the-effect-ofmusic-on-heart-rate. .
}

specific activities and tracking based on heart rate. Its function lies in mapping specifically-curated music (pre-processed) to the heartbeat intervals of the user. This is done through a combination of technologies and algorithms, namely a Joint Classification Regression Neural Network, connection to APIs, privacy and data security methods and database operations.

The application will be a Hybrid, allowing it to be used across platforms and developed for Android and iOS users and a Web App. The app will be integrated with wearables compatible with Android and iOS devices. The sending and syncing of data for Android devices will be facilitated through the Wearable Data Layer API, the Google Play services. Similarly, for iOS devices, the Healthkit, provided by Apple, will be used to access the heartbeat data collected and fed to the algorithm. Data privacy is of the utmost importance during this process and will be secured as per the Health Insurance Portability and Accountability Act (HIPAA) rules. Through a combination of encryption protocols and differential privacy, the healthcare data results from the classification model and results from the regression model will be secured. The web app serves the function of a data portal for 
the user to track the data interactions between their wearables and the app.

The application, across all platforms, requires internet access for access to the extensive music repository. The repository is then processed through the classification algorithm, sorted by genre and activity, and the results are provided to the regression algorithm.

The essential features of the app are:

1. Multi-OS Support: The Heartune App is supported on multiple platforms, and sessions data from one device can be viewed on another device or platform when connected to an account.

2. Pairing with Wearables: The Heartune App can pair with wearables based on the user's platform based on the existing paired devices associated with the mobile device being used. (i.e. The app will request permission to use health data generated by a user's Apple Watch when it is paired with an $\mathrm{iOS}$ device.)

3. Sending and Syncing data with Wearables and Paired Devices: Required data (as per the agreedupon permissions) generated by the wearable device and the paired devices will be sent when the user is using the app/has allowed the app to run in the background process. The results from the Heartune app will be sent back to the wearables through the API. Session results will also be shared and stored in a database associated with the user's account to view the web application.

4. Selection of Genre and Activity: The Heartune App allows the user to choose from a list of activities and genres which will be used as parameters for the classification algorithm. The application will then use the results of the classification algorithm to create a pre-processed list of music which will be sent to the regression algorithm to be mapped to the heartbeat intervals.

5. Tracking Heartbeat Intervals: The application has a live display of the user's heartbeat at the time of using the application with indicators if the heartbeat is within standard or abnormal range for the activity selected.

6. Detection of irregular Heartbeat Intervals: The application will alert the user if the heartbeat is in an abnormal range for the activity. If SOS permissions have been enabled and the heartbeat is in an 


\section{VOLUME: 13, JANUARY-JUNE 2022}

abnormal range for a prolonged period of time, the SOS functionality of the wearable may be activated, and the necessary emergency contacts will be contacted.

7. Adjusting Type and Tempo of Music to Relax or Elevate Heartbeat: When the heartbeat is in an abnormal range as per the activity selected, the app will attempt to regulate the heartbeat by adjusting the type and tempo of the music being played to either elevate or relax the heartbeat.

8. Display of Visual Graphic representing Music Sound-waves corresponding to Heartbeat Intervals: A visual graphic showcasing the heartbeat and the movement of music sound waves will be displayed for aesthetic appeal and to see movements in the frequency of sound waves.

9. Session Summaries: Session summaries of the songs played, the average heartbeat and other health metrics are generated and displayed for the user to view. The data is also available for view on the web application, corresponding to the logged-in user.
The application is powered by a model that utilizes two classification algorithms and a regression model that performs the functions sequentially. Alternatively, a multi-output model could be utilized. The model utilizes deep learning and libraries such as TensorFlow and Keras. The prediction problems surfaced by the application require both numeric and class label values for each input.

The essential features of the model are:

1. Classification of ECG-based heartbeat data: Given that ECG signal processing and heartbeat segmentation are conducted by the wearable device itself, the classification algorithm does not attempt to process or segment the heartbeat intervals. Instead, the data received from the wearables are classified into normal and abnormal ranges as per the standards outlined by the Association for the Advancement of Medical Instrumentation (AAMI). Given that this requires individualized data from the user, this is a local task and is unique to the device.

2. Classification of Music Dataset to Genre/Activity: The model can group music based on genre and mood through Deep Learning, 


\section{VOLUME: 13, JANUARY-JUNE 2022}

Transfer Learning and TensorFlow.

During the transfer learning process, features are extracted using the base layers of the pretrained model and the last layer is replaced by a custom classifier. Through the combination of pooling layers and 1D convolutional layers, key features can be extracted from the raw audio, and the genre and mood can be classified. The genre and mood are run through a separate classification algorithm to map it to the activity. This is run as a global task and is not unique for the user or device.

3. Regression of results from classification: A Deep Neural Network was utilized to map the pre-processed music dataset to the segmented heartbeat intervals and monitored at fixed intervals.

4. Self-Learning and Feedback: The results from the classification algorithms are fed back to improve the efficacy and speed of classification to increase the accuracy of the model over time.

The front end of the application will be designed to increase usability, efficacy and aesthetic appeal, following a consistent colour scheme as shown in Fig (ref below). The user will have the option to use the application in Dark, Light or Dynamic mode. (Dynamic mode would alter the colour of the background (Dark or Light) as per the time of day (additional permissions to access location would be required for this feature). 


\section{VOLUME: 13, JANUARY-JUNE 2022}

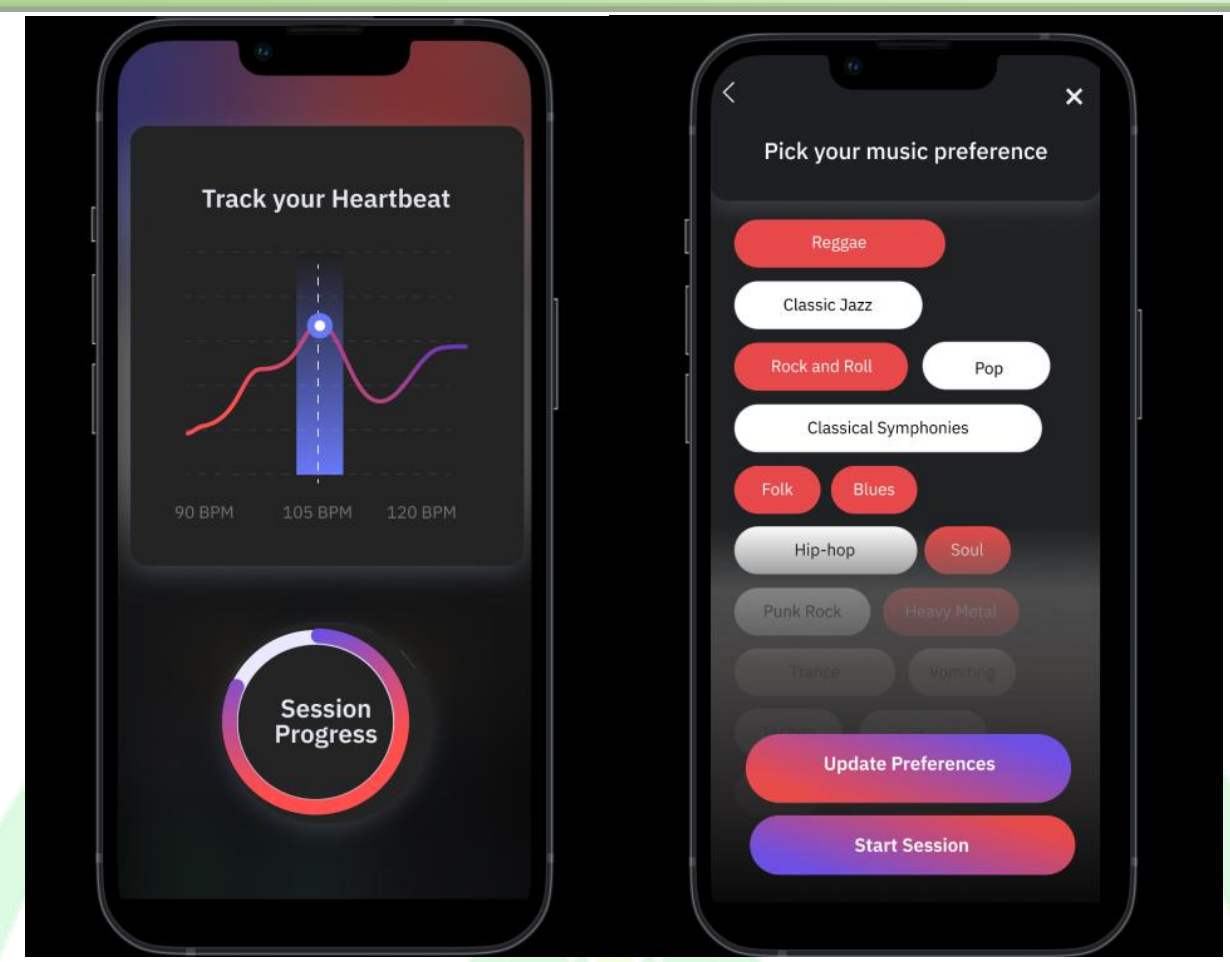

User Interface Mockups generated by Ishaan Singh, using Figma

\section{RESEARCH ANALYSIS}

To determine the efficacy of the application and its medical-grade benefits, the following section would further analyse a set of over 50 research works, meta-analyses and experimentations conducted regarding music therapy and several medical disorders. These studies would enable the application's viability to be tested and corroborate future uses of the application in collaboration with more doctors and therapists. The research analysis is hence evaluative of the various studies' methods and tools of analysis; this hence would determine the possible integration of Heartune as a supplementary treatment to the following diseases.
Working with both old and recent studies, the analysis would cater to a wider audience and determine the target audience for the application. With the evaluation of several successful methods of administration of music therapy for the supplemental treatment of Autism, Alzheimer's, Depression and other developmental disorders that majorly impact infants, the analysis would thereby be utilised to determine the timeline for the application as well as its growth strategies within the upcoming financial year. 


\section{Heartune and Autism}

${ }^{7}$ Autism Spectrum Disorder (ASD), a developmental disorder, affects a person's ability to communicate with symptoms like lack of eye contact, repeating words or phrases, and voice modulation. ${ }^{8}$ About 1 in 44 children have been identified with ASD according to the estimates from CDC's Autism and Developmental Disabilities Monitoring (ADDM) network. There are 5 types of Autism Spectrum Disorders: Asperger's syndrome, Rett syndrome, Childhood Disintegrative Disorder, Kanner's Syndrome, and Pervasive Developmental Disorder. Diagnosing a child with Autism is a difficult task due to the qualitative way of testing. Doctors usually look at a child's developmental history and behaviour in order to make a diagnosis ${ }^{9}$. For instance, the medical professional might ask whether the baby starts to mimic sounds and facial expressions by 9 months. Surprisingly, despite the high prevalence of ASD, the causes of the disease aren't completely understood. However, it is understood that

\footnotetext{
7"What Is Autism?" WebMD, WebMD, Apr. 2002,

www.webmd.com/brain/autism/understanding-autism-basics.

8 CDC. "Data \& Statistics on Autism Spectrum Disorder."

Centers for Disease Control and Prevention, 2 Dec. 2021, www.cdc.gov/ncbddd/autism/data.html.

9 CDC. "Screening and Diagnosis of Autism Spectrum Disorder." Centers for Disease Control and Prevention, 13 Mar. 2020,

www.cdc.gov/ncbddd/autism/screening.html\#: :text=Diagnosin g\%20autism\%20spectrum\%20disorder\%20(ASD, at $\% 2018 \% 20$ months\%20or\%20younger.. .
}

doctors like genetic mutations, environment, metabolic metations, and maternal history of viral infections can play a role in causing the disease ${ }^{10}$.

The most effective treatments available for ASDs are applied behavioral analysis (ABA), occupational therapy, speech therapy, physical therapy, and pharmacological therapy.

Some of the treatments of ASDs can be listed below:

- Behaviour and Communication Approaches: Behavioral and communication therapies are potential treatments for various ASDs. A combination of Behavioral and communication therapies are used because autistic children face behavioral issues even if they learn how to communicate. Children who understand how to behave in different environments and situations in addition to communicating find it easier to indulge in social interactions ${ }^{11}$.

\footnotetext{
${ }^{10}$ Cherney, Kristeen. "Everything You Need to Know about Autism Spectrum Disorder (ASD)." Healthline, Healthline Media, 3 Nov. 2021, www.healthline.com/health/autism\#causes.

11 "Behavioral and Communication Approaches." Mentalhelp.net, 2015, www.mentalhelp.net/autism/behavioraland-communication-approaches/. .
} 
- Cognitive Behaviour Therapy: ${ }^{12}$ Cognitive behaviour therapy focuses on the connection between thoughts, feelings and behaviour. Patients learn to deal with their feelings and control their behaviour in various situations.

- Medication: There are currently no medications that can cure ${ }^{13}$ ASDs, however, some medications can control some of the symptoms of the disease. For instance, Tricyclics can be used to treat compulsive behaviour and depression.

Despite the availability of following treatments, there are no proven treatments for Autism Spectrum Disorders till date ${ }^{14}$. However, music therapy (MT) has the potential to be useful in the treatment of ASDs. A study by ${ }^{15}$ Geetha Bharati et. al

12 "'Cognitive Behavior Therapy for Autism."

Https://Www.nichd.nih.gov/, 31 Jan. 2017,

www.nichd.nih.gov/health/topics/autism/conditioninfo/treatment s/cognitive-behavior. .

13 "Medication Treatment for Autism."

Https://Www.nichd.nih.gov/, 19 Apr. 2021,

www.nichd.nih.gov/health/topics/autism/conditioninfo/treatment s/medication-treatment.

14 CDC. "Treatment and Intervention Services for Autism Spectrum Disorder." Centers for Disease Control and Prevention, 23 Sept. 2019,

www.cdc.gov/ncbddd/autism/treatment.html\#: :text=Currently $\% 2 \mathrm{C} \% 20$ no $\% 20$ treatment $\% 20$ has $\% 20$ been,community $\% 20 \% 5 \mathrm{~B}$ $1 \% 2 \mathrm{D} 6 \% 5 \mathrm{D}$..

${ }^{15}$ Bharathi, Geetha, et al. "Music Therapy as a Therapeutic Tool in Improving the Social Skills of Autistic Children." The Egyptian Journal of Neurology, Psychiatry and Neurosurgery, vol. 55, no. 1, 25 June 2019 ,

ejnpn.springeropen.com/articles/10.1186/s41983-019-0091-x, 10.1186/s41983-019-0091-x. . investigated the effect of music therapy on 60 children (30 girls and 30 boys) ranging from the age of 6-12 years by studying their pre and post therapy CARS score. The social skills of the children of the control and MT were measured using the TSSA as the pre-test.The MT group was divided into active and passive MT groups, in which the active group were involved in activities like singing, dancing, and playing instruments and the passive groups were involved in listening to recorded music tracks without any kind of interactions. The results showed that there was an improvement in the social skills rating in both active and passive groups of ASD children. There was an increase of 74.08 to 114.85 in the social skills rating for the active group and 72.31 to 112.62 for the passive group. Whilst the active group showed a much greater improvement in the 'Understanding taking' ability, there were similar improvements in the 'initiates interaction' and 'maintains interaction' for both groups. ${ }^{16}$ Children with ASD show a heightened interest and response to music making it a reliable therapeutic tool. Outcomes from the use of music as a therapeutic intervention with children along the autism spectrum disorder

\footnotetext{
${ }^{16}$ American Music Therapy Association. Music Therapy as a Treatment Modality for Autism Spectrum Disorders. 2012.
} 


\section{VOLUME: 13, JANUARY-JUNE 2022}

includes improvement in interpersonal skills, social skills, and cognitive skills ${ }^{17}$. According to clinical trials, Music is a universally appealing medium. It acts as a link between people and their surroundings in a non-threatening environment, facilitating relationships, learning, selfexpression, and communication. Music has the ability to capture and hold one's attention. It may be utilized as a natural "reinforcer" for desired reactions as it is very motivating.

When observing the correlation of Autism and music therapy, a reference to the work of Brown $^{18}$ In her analysis of the effectiveness of music therapy and autism. In her analysis of music therapy's effectiveness and correlation with autism, she elaborates on four major causes of the high efficacy rate of the supplemental treatment. The primary reasons describe the fostering of creative thoughts and social behaviour in children, primarily due to the characteristic nature of music. These reasons are complemented by her description of the close link music has to human emotions and by referring to

17 Dawn De Vries, et al. "Music as a Therapeutic Intervention with Autism: A Systematic Review of the Literature." Therapeutic Recreation Journal, vol. 49, no. 3, 2015, js.sagamorepub.com/trj/article/view/6085.

${ }^{18}$ Brown, Sandra M.K. "Autism and Music Therapy Is Change Possible, and Why Music?" Journal of British Music Therapy, vol. 8, no. 1, June 1994, pp. 15-25, journals.sagepub.com/doi/abs/10.1177/13594575940080 $0105,10.1177 / 135945759400800105$. several case studies of children (Mary, George, David, Ben and Andy) who have benefitted from this specific treatment. From activating the children's emotional states to developing their cognitive and social skills, the treatment was proven to be significantly beneficial for the test subjects.

Moreover, such a study further corroborates utilising the application 'Heartune' to lead to such development in children. Based on the results derived, Heartune serves to administer such music therapy based on the requirement of the patient and the advice given by a given healthcare professional. Such supplementary treatment would passively lead to the betterment of the child's cognitive, physical and emotional state of mind.

This makes passive music therapy a potential supplement treatment for Autism through Heartune, alongside the aforementioned treatments. As the other therapies focus on improving the behavioural and cognitive skills of the patient, passive music therapy will aid the treatment to work in a shorter duration of time. 


\section{$\underline{\text { Heartune and Alzheimer's }}$}

${ }^{19}$ Alzheimer's disease is a neurodegenerative disease which causes atrophy and is the most common cause of Dementia. Aside from other factors, age plays a major major role in causing Alzheimer's disease, as after turning 65 the risk of Alzheimer's disease doubles every 5 years. At the age of 85 , it nearly becomes one third. There are 2 major proteins which can are hypothesised to cause Alzheimer's disease:

\section{Tau Proteins}

${ }^{20}$ The Tau protein has the function of stabilizing the internal microtubules which are long cylindrical structures controlling cell division, intracellular transport, and the shape of the cells. ${ }^{21}$ In Alzheimer's disease abnormal chemical changes take place in the tau tables which cause them to detach from microtubules and stick to other tau molecules which form neurofibrillary tangles, harming the synaptic communication of neurons.

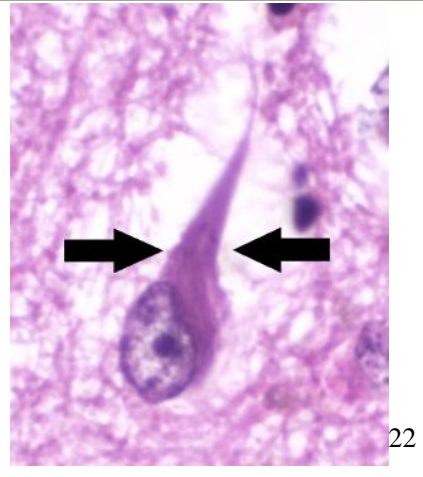

\section{Amyloid-Beta Plaques}

${ }^{23}$ Amyloid plaques form when pieces of protein called beta-amyloid aggregate are produced when a much larger amyloid precursor protein (APP) is broken down composed of 771 amino acids. ${ }^{24}$ Even Though, Amyloid Beta plaques are a hallmark of Alzheimer's disease it is still not known how they damage the nervous system. It is hypothesised that the protein breaks into fragments and releases free radicals, which affect neurons or they might form tiny holes in neuronal membranes which lead to an unregulated influx of calcium that can cause neuronal death.
19 ،6 Alzheimer's Disease - Symptoms and Causes." Mayo

Clinic, 2021, www.mayoclinic.org/diseases-

conditions/alzheimers-disease/symptoms-causes/syc-20350447.

20 "Tau Protein and Alzheimer's Disease: What's the Connection? | BrightFocus Foundation.” Brightfocus.org, 2021, www.brightfocus.org/alzheimers/article/tau-protein-andalzheimers-disease-whats-connection.

${ }^{21}$ https://www.facebook.com/NIHAging. "What Happens to the Brain in Alzheimer's Disease?" National Institute on Aging, 2017, www.nia.nih.gov/health/what-happens-brain-alzheimersdisease\#: :text=In\%20Alzheimer's\%20disease $\% 2 \mathrm{C} \% 20$ howeve r\%2C\%20abnormal,the\%20synaptic\%20communication\%20bet ween $\% 20$ neurons..

\footnotetext{
22 Wikipedia Contributors. "Neurofibrillary Tangle." Wikipedia, Wikimedia Foundation, 14 Dec. 2021, en.wikipedia.org/wiki/Neurofibrillary_tangle.

23 News-Medical. "What Are Amyloid Plaques?" NewsMedical.net, 17 Jan. 2014, www.news-medical.net/health/Whatare-Amyloid-Plaques.aspx. .

${ }^{24}$ One, Communications. Beta-Amyloid and the Amyloid Hypothesis. 2008
} 


\section{VOLUME: 13, JANUARY-JUNE 2022}

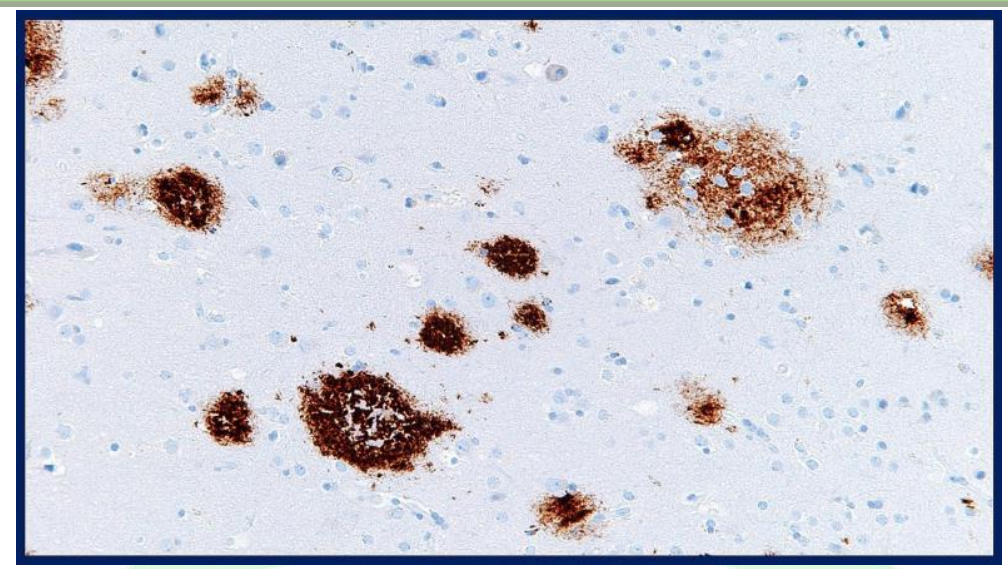

Alzheimer's. It's taken as a pill once a day

There are certain medications which are given to Alzheimer's disease. They include:

\section{Cholinesterase inhibitors}

One way Alzheimer's disease harms the brain is decreasing the level of acetylcholine important for alertness, memory, thought and judgement. These inhibitors increase the amount of acetylcholine in the brain and also prevents its breakdown. They cant reverse or stop Alzheimer's disease. These become useless in later stages.

Donepezil (Aricept) is approved to treat all stages of the disease. It's taken once a day as a pill.

Galantamine (Razadyne) is approved to treat mild to moderate

\footnotetext{
25 “Amyloid Beta Plaques - Google Search." Google.com, 2011, www.google.com/search?q=amyloid+beta+plaques\&rlz=1C5C HFA_enIN914IN914\&sxsrf=AOaemvLPLB8G3fke2A3CDWo B8T31r9WORw:1640420548251\&source $=1 n m s \&$ tbm $=i$ sch\&sa $=$ X\&ved=2ahUKEwj42r3gwv70AhVn6XMBHfK0C3UQ_AUoA XoECAIQAw\#imgrc=PQK_LwH0K9-pBM
} or as an extended-release capsule twice a day.

Rivastigmine (Exelon) is approved for mild to moderate Alzheimer's disease. It's taken as a pill. A skin patch is available that can also be used to treat severe Alzheimer's disease.

\section{Memantine}

Memantine (Namenda) is approved by the FDA for the treatment of moderate to severe Alzheimer's disease. It works by regulating the activity of glutamate, a messenger chemical widely involved in brain functions - including learning and memory. It's taken as a pill or syrup. Common side effects include dizziness, headache, confusion and agitation. The FDA has also approved a combination of donepezil and memantine (Namzaric), which is taken as a capsule. Side effects include headache, dizziness, nausea and diarrhoea. 
${ }^{26}$ Amongst these medications, which can positively impact Alzheimer's patients, Various studies have shown that music therapy can slow the progression of Alzheimer's in patients. A case study by Johnson and colleagues, in which the subjects were 74-year-old monozygotic twins with no family history of dementia in which one twin had Alzheimer's disease, and the other did not have Alzheimer's disease, both having 14 years of education. The mini-mental state exam score of the AD twin was 23 (mild dementia), and the score of the NC (for anonymity) twin was 27. To analyse the data, the PF\&C (Paper fold and cutting task) scores were analysed before and after three sessions of listening to a piece by Mozart. For the $\mathrm{AD}$ twin, when the pre and post-test scores of the sessions were analysed, a remarkable improvement was seen in the scores of an average of $3.67 / 8$ points, showing improvement in spatial-temporal reasoning. On the second day of testing, the post-test score again improved. Thus, this improvement was observed across all Mozart listening testing sessions and with two PF\&C sets. No improvements, however, were observed in the post-test

\footnotetext{
26 Julene Johnson, Carl Cotman, Cora Tasaki \& Gordon Sha (1998) Enhancement of spatial-temporal reasoning after a Mozart listening condition in Alzheimer's disease: A case study, Neurological Research, 20:8, 666-672, DOI:
}

scores obtained by the AD twin following all of the control conditions. Therefore, this study acts as supporting evidence for the efficacy of music therapy. Using Heartune to play tracks like the Mozart tracks in the above case study can improve skills like spatial-temporal skills.

A clinical trial conducted by Sakamoto and colleagues recruited 127 dementia patients, from which 39 met the eligibility criteria. In the study, three groups (Nonintervention Control, Passive music intervention and Interactive music intervention) were compared in which each intervention was performed for 30 minutes once a week for ten weeks. In the Control group, a caregiver and music provider observed participants from a distance without directly interacting with them and any music intervention. The Passive group participants passively listened to the selected music via a CD player, which means there was no interaction like singing along or dancing. In the Interactive music intervention, the session was conducted individually by a music facilitator who directly interacted with each participant like singing along, dancing, and clapping. The music facilitators watched the participants before intervention and determined each participant's level of cognitive function and Behavioral and Psychological 
Symptoms of Dementia (BPSD)

characteristics. In the Passive group, the comparison of the autonomic nervous system indices. The autonomic nervous system is a control system that acts largely unconsciously and regulates bodily functions, such as the heart rate, digestion, respiratory rate, pupillary response, urination, and sexual arousal. Before and after music intervention, - the parasympathetic nerve activity (The parasympathetic nervous system is one of three divisions of the autonomic nervous system) became dominant. This was also seen in the interactive group, but there was no significant increase in parasympathetic nerve activity. For the long term, which is after ten intervention sessions for the Passive groups, the patients' phobias were reduced. In the Interactive group, affective disturbance, anxieties and phobias, paranoid and delusional ideation, and the global rating (which indicates caregiver burden) were reduced. In the control group, activity disturbance and affectivity disturbance were increased. In contrast, three weeks after music intervention, BPSD had significantly increased in both the Passive and Interactive groups after the sessions had ceased. The Control group exhibited no changes in BPSD at three weeks after the intervention ${ }^{27}$.

\footnotetext{
${ }^{27}$ Sakamoto, Mayumi, Hiroshi Ando, and Akimitsu Tsutou.
}

Overall, it can be concluded that this implementation of passive music therapy as a supplemental treatment for Alzheimer's proves to be highly beneficial, especially to older patients. Moreover, due to the inherent amnesic properties of Alzheimer's, a regulated and automated form of music therapy is required to ensure that the patient does slowly recover. Hence, Heartune serves to be extremely practical in the case of reducing the impact that Alzheimer's has on the patients' lives. Moreover, with the application's features, the supplementary treatment would become more streamlined and thus be highly beneficial for the patients.

\section{Heartune and Depression}

${ }^{28}$ Depression is a mood disorder causing a persistent sadness or loss of interest. It can be classified into eight types:

1. Anxious distress is a kind of depression marked by unusual restlessness or concern about upcoming events, or a sense of loss of control.

2. Anxious distress is a kind of depression marked by

2013. "Comparing the Effects of Different Individualized Music Interventions for Elderly Individuals with Severe Dementia." International Psychogeriatrics 25 (5): 775-84. https://doi.org/10.1017/s1041610212002256

\footnotetext{
28 "Depression (Major Depressive Disorder) - Symptoms and Causes." Mayo Clinic, 2018, www.mayoclinic.org/diseasesconditions/depression/symptoms-causes/syc-20356007.
} 
extraordinary restlessness or

concern about upcoming events, or a sense of loss of control.

3. Mixed features - depression and mania simultaneously, with higher self-esteem, excessive talking, and increased activity.

4. Melancholic features- Extreme depression, a lack of response to something that used to offer pleasure, associated with a worsening mood in the morning, dramatic changes in appetite, feelings of guilt, agitation, or sluggishness, and feelings of guilt, agitation, or sluggishness.

5. Atypical features- The capacity to be temporarily cheered by pleasant occurrences, increased appetite, an excessive desire for sleep, rejection sensitivity, and a heavy sensation in the arms or legs.

6. Psychotic features- Depression accompanied by delusions or hallucinations, including feelings of inadequacy or other unpleasant themes.

7. Catatonia is a depression characterised by uncontrolled and purposeless movement or rigid and inflexible posture.

8. Depression that develops during pregnancy or in the weeks or months following birth is known as peripartum

depression

(postpartum)

9. Seasonal pattern - sadness linked to seasonal variations and less solar exposure.

${ }^{29}$ In 2011, Finnish researchers analysed 79 people with depression between 18 and 50 in the British Journal of Psychiatrist. According to the Central Finland Health Care District (where the study was done), the standard care was given to 46 participants, which included five to six psychotherapy sessions, antidepressants, and psychiatric counselling, according to the Central Finland Health Care District (where the study was done). The remaining 33 individuals received the same conventional treatment as the others. Twenty individuals received 60-minute biweekly music therapy sessions. The sessions used a variety of musical instruments, including a mallet instrument, a percussion instrument, and an acoustic djembe drum, to express themselves musically. All improvisations were recorded for future processing and discussion, and the therapist and the client used the same instruments. At baseline, three months after the intervention, and six

\footnotetext{
${ }^{29}$ Borchard, Therese. "How Music Therapy Can Relieve Depression." EverydayHealth.com, 4 May 2017, www.everydayhealth.com/columns/therese-borchard-sanitybreak/music-therapy-to-relieve-depression/.
} 
months after the intervention, depression ratings were evaluated. After three months, individuals who got music therapy in addition to conventional care had much less depressive symptoms than those who just received standard care. In addition, general performance increased. Therefore, this study acts as supporting evidence for the efficacy of music therapy for treating depression.

Moreover, whilst explicitly looking at the experimentation conducted by Sonia et al., it can be seen that this correlation between music therapy and depression further extends a comparison with other forms of treatment of depression. In the work cited, the researchers took a set of randomized control trials and controlled clinical trials wherein they could compare the usual treatment with the supplementary treatment. In almost all cases, their results indicated a significant elevation in mood post the administration of music therapy, thereby corroborating the purpose of the application. Moreover, their results indicate that music therapy must be complemented with treatment as usual to have maximum effects. However, it must be noted that this supplementary treatment has only been proven to be effective for mild depression.
When used with the prescription of a therapist or based on the in built features of the application, the user can listen to a myriad of music options based on their current heartbeat. When complimenting a slow heartbeat with gradual increments in the bpm, there can be a significant elevation in the mood of the consumers and hence the major symptoms for depression can fade in the short run. As consistent music therapy is required to make a more impressionable impact, the application could be providing such therapy on a daily basis, thereby supplementing the other treatment of the disease.

\section{$\underline{\text { Heartune and Developmental Disorders }}$}

Developmental Disorders as defined by the Institute of Medicine (US) Committee on Nervous System Disorders in Developing Countries $^{30}$ is a condition causing the restriction of ability of a given person due to specific issues regarding their nervous system. Primarily detected early in childhood and infancy with delayed developmental milestones, these developmental disorders are visible in infants that have delayed development of Fine motor skills, gross motor skills, 'play'

\footnotetext{
30 "Neurological, Psychiatric, and Developmental Disorders." Neurological, Psychiatric, and Developmental Disorders: Meeting the Challenge in the Developing World., 2001, doi:10.17226/10111.
} 


\section{VOLUME: 13, JANUARY-JUNE 2022}

skills and feeding/eating skills alongside a recent inclusion of prolonged screen time that becomes a key factor in the damaging of the nervous system of young infants.
Usually measured from ages 0-5, these indicators are further extended to detect the mental retardation of children as shown by the table below, taken from [34]:

Figure 1: ICD Codes for the various developmental disorders based on specific skill development delays ${ }^{31}$

\section{ICD-10 Code}

\begin{tabular}{l|l}
\hline Cognitive & \\
\hline Mental Retardation & F70 \\
\hline Mild (IQ approximately 50-69) & F71 \\
\hline Moderate (IQ approximately 35-49) & F72 \\
\hline Severe (IQ approximately 20-34) & F73 \\
\hline Profound (IQ below 20) & F81 \\
\hline Specific Learning Disabilities & F81.0 \\
\hline Reading (Dyslexia) & F81.2 \\
\hline Mathematics (Dyscalculia) & \\
\hline Other & \\
\hline Motor & G80 \\
\hline Cerebral Palsy & B91 \\
\hline Post-Polio Paralysis & G71.0 \\
\hline Muscular Dystrophies & Q05 \\
\hline Spina Bifida & G12 \\
\hline Spinal Muscular Atrophies & M01-M03. \\
\hline Other & Q65-Q79 \\
\hline & \\
\hline
\end{tabular}

\footnotetext{
${ }^{31}$ World Health Organization (WHO). ICD-10:International statistical classification of diseases and related health problems. 10th revision. World Health Organization: Geneva, 1992.
} 
VOLUME: 13, JANUARY-JUNE 2022

\begin{tabular}{|c|c|}
\hline \multicolumn{2}{|l|}{ Wrisiom } \\
\hline Refraction Disorders: & HS2 \\
\hline Cataract infantille and juvenile & 1126.0 \\
\hline Chorioretinal Inflammation, infectious or parasitic & H32.0 \\
\hline Nightblindmess, due to vitamin A deficiency & Eso.s \\
\hline Other & Q10-Q1S \\
\hline \multicolumn{2}{|l|}{ Fecoringe } \\
\hline Conductive and Semsorimeural & H9O \\
\hline Oiher & Q16 \\
\hline \multicolumn{2}{|l|}{ Flecuring and Speech } \\
\hline Specific Speceh Articulation Disorder & F 80.0 \\
\hline Expressive L anguage Disorder & F8O. 1 \\
\hline Receptive I anguage Disorder & $\mathbf{F} 80.2$ \\
\hline \multicolumn{2}{|l|}{ Befaction } \\
\hline Attention-Deficit Hyperaetivity Disorder & F90.O \\
\hline Pervasive Developmental Disorder, including autism & $\mathbf{F} 84$ \\
\hline Oiher & F8O-F98 \\
\hline
\end{tabular}

Hence, the main link between music therapy and these developmental disorders arises post their detection and as a significant part of their supplementary treatment. These disabilities may be motor, verbal/elocution, behavioural and cognitive as these are often linked back to the central nervous system and its dysfunction in their specific cases. The correlation between Music therapy and developmental disorders would be conducted by cross-examination of two major studies. The first analysis would be of the results [Montello] in comparing active and passive music therapy for the treatment of children with $\mathrm{ADD}$ (Attention Deficit Disorder), emotional instability, and delayed/slowed knowledge assimilation. The secondary analysis would be of the impact passive music therapy has on the socio-emotional skillset of students with ADHD (Attention Deficit Hyperactive Disorder $)^{32}$.

With the random distribution of children between 3 major classes defined by the following conditions - A(Receipt of active music therapy for 12 weeks followed by passive music therapy for 12 weeks), $\mathrm{B}$ (Receipt of passive music therapy for 12 weeks followed by active music therapy for 12 weeks) and $\mathrm{C}$ (a receipt of active music therapy for 24 weeks). The approximate 6-month long research study resulted in significant impacts on test groups $\mathrm{A}$ and $\mathrm{B}$ in particular wherein students experienced a drastic reduction in hostility in both and a considerable

\footnotetext{
${ }^{32}$ Rajendran, Renuchitra. "Effectiveness of Active and Passive Participation in Music Therapy on Social Emotional Skills of ADHD Children". International Journal of Science and Research (IJSR) volume 6. (2015): 2319-7064.
} 
reduction in lack of motivation and attention within test group B. With study group A, however, there was a noticeable increment in the ego development of the students, leading to the conclusive statements that active music therapy may not solely be used for the treatment of such disorders. An extension of the study further developed resolutions that indicated the ability of test group B students to participate in group activities with more ease as compared to test group A, illustrating a drastic improvement in communication and interpersonal skills.

Moreover, the second study utilizes the Vanderbilt ADHD diagnostic teacher rating scale and the teacher's report form to determine the intellectual competence, anxiety levels, depression levels, situational functioning, social reasoning, inattention levels and hyperactivity levels. Using 30 students with 15 respectively in control and experimental groups, the researchers conducted these two tests before and after the administration of active and passive music therapy for the experimental group to find significant reductions in difficulties developing socioemotional skills for the sets of students that engaged in passive and active music therapy as compared to their control counterparts. Hence the conclusion of the paper stated that for children in the developmental stages - infancy to preadolescence - the utilization of music therapy would be highly beneficial for corrections in any socio-emotional skills of the child.

Thus, the admission of passive music therapy based on treatments prescribed by music therapists, cardiologists, developmental paediatricians, and others is proven to enable corrections within the dysfunctional nervous system of children suffering from developmental disorders. Due to the developing nervous system of children, they are more prone to have altercations within their system during which Heartune could be utilised as a method of transmission of the treatment. The therapeutic abilities of the application could potentially be merged with prescribed treatments from doctors and therapists to begin long term treatments of patients from ages 0-12 specifically. Moreover, passive music therapy with Heartune could also be utilised for adults battling these disorders; however, its efficacy in adults is yet to be proven.

As Heartune primarily relies on the fundamental concepts of passive music therapy to deliver a supplementary 
treatment to its clients, the application's viability would once again be verified through this examination of the efficacy of passive music therapy in the case of these two studies. Moreover, the application could potentially be used with the advice of a certified doctor to administer passive music therapy. By using the doctor's inputs, the application would utilise the patient's heartbeat and minimise volatility and erratic shifts in the heart rate by maintaining a specific tempo of the music. Moreover, the application would administer this music therapy based on the time, type and duration of the therapy on the given device.

\section{EVALUATION}

The fundamental correlation upon which the notions that empower Heartune are built are demonstrated through the works of Cervellin et $\mathrm{Al}^{33}$ in their analysis of the impact music beats have on the listeners heart and heart rate. This hence proves the potential efficacy of the application for its fundamental purpose of connecting the heart rate of its user to the music played or the music played to the heart rate of its

\footnotetext{
${ }^{33}$ Cervellin, Gianfranco, and Giuseppe Lippi. "From Music-Beat to Heart-Beat: A Journey in the Complex Interactions between Music, Brain and Heart." European Journal of InternalMedicine, vol. 22, no. 4, Aug. 2011, pp. 371-374, 10.1016/j.ejim.2011.02.019.
}

user. The bidirectional nature of the signalling mechanism followed by the heart with music is essential for the application's success.

Based on the research studies previously cited, there is a significant advantage seen with the implementation of passive music therapy as a supplementary treatment for Alzheimer's, ADHD, Autism and Developmental disorders. With the majority of papers centring around infants, children and adolescents, the target group for Heartune can thus be recognized as both adolescents and parents that would benefit from the utilization of the application. Moreover, the studies state the importance of utilizing music therapy and its advantages to the members within the experimentation group.

\section{CONCLUSION \\ RECOMMENDATIONS}

AND

Based on the analysis conducted, the efficacy and necessity of an application such as 'Heartune' may be verified as a supplementary treatment for patients primarily infants, children and adolescents - suffering from Alzheimer's, Autism, Depression and other developmental disorders. By implementing passive music therapy whilst using the patient's heartbeat, Heartune would hence benefit 
several patients and significantly decrease the impacts and severity of their condition.

With the several research works previously cited and evaluated; the recommendations made by the Heartune team would revolve around the prescribed utilisation of the application. The application is a supplemental treatment and does not entail diagnosis, complete treatment, curing or eradicating the illness. However, the application would be significantly beneficial to over $17 \%^{34}$ of the student's within the United States that are already battling developmental disorders.

\section{REFERENCES}

[1] Tuning in: How music may affect your heart - Harvard Health "Tuning In: How Music May Affect Your Heart - Harvard Health". Harvard Health, 2018,

https://www.health.harvard.edu/hearthealth/tuning-in-how-music-may-affectyour-heart

[2] S., Suguna, and Deepika K. "The Effects of Music on Pulse Rate and Blood Pressure in Healthy Young Adults, International Journal of Research in Medical Sciences, vol. 5, no. 12, 2017, p. 5268.

${ }^{34}$ CDC. "CDC's Work on Developmental Disabilities." Centers for Disease Control and Prevention, 25 Sept. 2019, www.cdc.gov/ncbddd/developmentaldisabi lities/about.html. doi:10.18203/2320-6012.irms20175438.

[3] "What Is Music Therapy?" Taking Charge of Your Health \& Wellbeing,

www.takingcharge.csh.umn.edu/commonquestions/what-music-therapy.

[4] Montello, L., and E. E. Coons. "Effects of Active versus Passive Group Music Therapy on Preadolescents with Emotional, Learning, and Behavioral Disorders." Journal of Music Therapy, vol. 35, no. 1, 1998, pp. 49-67., doi:10.1093/jmt/35.1.49.

[5] Wagner, Gabriela. "The BENENZON Model of Music Therapy." Nordic Journal of Music Therapy, vol. 16, no. 2, 2007, pp. 146-147., doi:10.1080/08098130709478184.

[6] oakesril, Hayes School of Music."Hayes School of Music.' " The Bonny Method of Guided Imagery and Music, $24 \quad$ Sept. 2021, music.appstate.edu/academics/specialprograms/bonny-method.

[7] IWANAGA, MAKOTO. "RELATIONSHIP between HEART RATE and PREFERENCE for TEMPO of MUSIC." Perceptual and Motor Skills, vol. 81, no. 2, Oct. 1995, pp. 435_440, pubmed.ncbi.nlm.nih.gov/8570336/, 10.2466/pms.1995.81.2.435.

[8] JEI. "The Effect of Music on Heart Rate | Journal of Emerging Investigators." Emerginginvestigators.org, 2013, emerginginvestigators.org/articles/theeffect-of-music-on-heart-rate. .

[9] What Is Autism?" WebMD, WebMD, Apr. 2002, 
www.webmd.com/brain/autism/understand ing-autism-basics. Accessed 18 Dec. 2021.

[10] CDC. "Data \& Statistics on Autism Spectrum Disorder." Centers for Disease Control and Prevention, 2 Dec. 2021, www.cdc.gov/ncbddd/autism/data.html.

Accessed 18 Dec. 2021.

[11] CDC. "Screening and Diagnosis of Autism Spectrum Disorder." Centers for Disease Control and Prevention, 13 Mar. 2020 ,

www.cdc.gov/ncbddd/autism/screening.ht $\mathrm{ml}$ : text-

Diagnosing\%20autism\%20spectrum\%20di sorder\%20(ASD, at\%2018\%20months\%20 or\%20

[12] pringer oN, KRIseO) * Pro aRting jou Miced 10 Know about A wism Spotrum Disturder (A5D) - Alcalthline, Healithline Media, 3 Nor 2021, www.healthline.com/health/autism\#causes . Accessed 18 Dec. 2021.

[13] "Behavioral and Communication Approaches." Mentalhelp.net, 2015,

www.mentalhelp.net/autism/behavioraland-communication-approaches/..

[14] "Cognitive Behavior Therapy for Autism." Https://Www.nichd.nih.gov/,31 Jan. 2017,

www.nichd.nih.gov/health/topics/autism/c onditioninfo/treatments/cognitivebehavior. .

[15] Medication Treatment for Autism." Https://Www.nichd.nih.gov/,19 Apr.2021, www.nichd.nih.gov/health/topics/autism/c onditioninfo/treatments/medicationtreatment. .

[16] CDC. "Treatment and Intervention Services for Autism Spectrum Disorder." Centers for Disease Control and Prevention, 23 Sept. 2019, www.cdc.gov/ncbddd/autism/treatment.ht ml\#: \#text=Currently\%2C\%20no\%20treat ment $\% 20$ has $\% 20$ been,community $\% 20 \% 5$ B1\%2D6\%5D.

[17] Bharathi, Geetha, et al. "Music Therapy as a Therapeutic Tool in Improving the Social Skills of Autistic Children." The Egyptian Journal of Neurology, Psychiatry and Neurosurgery, vol. 55, no. 1, 25 June 2019, ejnpn.springeropen.com/articles/10.1186/s 41983-019-0091-x, 10.1186/s41983-0190091-x. .

[18] American Music Therapy Association. Music Therapy as a Treatment Modality for Autism Spectrum Disorders. 2012.

[19] Dawn De Vries, et al. "Music as a Therapeutic Intervention with Autism: A Systematic Review of the Literature." Therapeutic Recreation Journal, vol. 49, no. 3 2015, js.sagamorepub.com/trj/article/view/6085.

[20] Brown, Sandra M.K. "Autism and Music Therapy - Is Change Possible, and Why Music?" Journal of British Music Therapy, vol. 8, no. 1, June 1994, pp. 1525 ,

journals.sagepub.com/doi/abs/10.1177/135 945759400800105, $10.1177 / 135945759400800105$. 
[21] "Alzheimer's Disease - Symptoms and Causes." Mayo Clinic, 2021,

www.mayoclinic.org/diseases-

conditions/alzheimers-disease/symptomscauses/syi-20350447. .

[22] «Tau Protein and Alzheimer's Disease: What's the Connection? BrightFocus Foundation." Brightfocus.org, 2021,

www.brightfocus.org/alzheimers/article/ta u-protein-and-alzheimers-disease-whatsconnection. Accessed 24 Dec.2021.

[23]

https://www.facebook.com/NIHAging."W hat Happens to the Brain in Alzheimer's Disease?" National Institute on Aging, 2017, www.nia.nih.gov/health/whathappens-brain-alzheimers-

disease\#: text=In\%20Alzheimer's\%20dise ase $\% 2 \varnothing \% 20$ however\%2C\%20abnormal,th e\%20synaptic\%20communication\%20bet ween $\% 20$ neurons... .

[24] Wikipedia Contributors, "Neurofibrillary Tangle." Wikipedia, Wikimedia Foundation, 14 Dec. 2021, en. wikipedia.org/wiki/Neurofibrillary tangle. I

[25] News-Medical. "What Are Amyloid Plaques?" News-Medical.net, 17 Jan. 2014,

www.news-medical.net/health/What-are-

Amyloid-Plaques.aspx. .

[26] One, Communications. Beta-Amyloid and the Amyloid Hypothesis. 2008

[27] «Amyloid Beta Plaques - Google Search." Google.com, 2011, www.google.com/search?q=amyloid+beta +plaques\&riz=ICSCHFAenIN914IN914\& sxsrf-

AOaemvLPLB8G3fke2A3CDWoB8T31r9 WORw: 1640420548251\&source=Inms\&t bm $=i s c h \& s a=X \& v e d=2 a h U K E w ; 42 r 3 g w v$ 70AhVn6XMBHfKOC3UQ

AUoAXoECAIQAw\#imgre=PQKLwHOK 9-DBM.

[28] Julene Johnson, Carl Cotman, Cora Tasaki \& Gordon Sha (1998) Enhancement of spatial-temporal reasoning after a Mozart listening condition in Alzheimer's disease: A case study, Neurological Research, 20:8, 666672

[29] Sakamoto, Mayumi, Hiroshi Ando, and Akimitsu Tsutou, 2013. "Comparing the Effects of Different Individualized Music Interventions for Elderly Individuals with Severe Dementia." International Psychogeriatrics25 (5): 77584.

https://doi.org/10.1017/s10416102120022 56

[30] «Depression (Major Depressive Disorder) - Symptoms and Causes." Mayo Clinic, 2018,

www.mayoclinic.org/diseasesconditions/depression/symptomscauses/syc-20356007. .

[31] Borchard, Therese. "How Music Therapy Can Relieve Depression." EverydayHealth.com, 4 May 2017,

www.everydayhealth.com/columns/therese -borchard-sanity-break/music-therapy-torelieve-depression/.Accessed 25 Dec.2021 
[32] Aalbers, Sonja, et al. "Music Therapy for Depression." Cochrane Database of Systematic Reviews, vol. 2017, no. 11, 16 Nov.

2017,www.cochranelibrary.com/cdsr/doi/1 0.1002/14651858.CD004517.pub3/full,10. 1002/14651858.cd004517.pub3. .

[33] Neurological, Psychiatric, and Developmental Disorders." Neurological, Psychiatric, and Developmental Disorders: Meeting the Challenge in the Developing World., 2001, doi:10.17226/10111.

[34] World Health Organization (WHO). ICD-10:International statistical classification of diseases and related health problems. 10th revision. World Health Organization: Geneva, 1992.

[35] Rajendran, Renuchitra. "Effectiveness of Active and Passive Participation in Music Therapy on Social-Emotional Skills of ADHD Children". International Journal of Science and Research (IJSR) volume 6. (2015): 2319-7064.

[36] Cervellin, Gianfranco, and Giuseppe Lippi. "From Music-Beat to Heart-Beat: A Journey in the Complex Interactions between Music, Brain and Heart." European Journal of internal medicine, vol. 22, no. 4, Aug. 2011, pp. 371-374, 10.1016/j.ejim.2011.02.019

[37] CDC. "CDC's Work on Developmental Disabilities." Centres for Disease Control and Prevention, 25 Sept. 2019,

www.cdc.gov/ncbddd/developmentaldisabi lities/about.html. 